\section{$\underset{\text { hommes }}{\text { \& migrations }}$}

\section{Hommes \& migrations}

Revue française de référence sur les dynamiques

migratoires

$1312 \mid 2015$

Diasporas iraniennes

\title{
Regards croisés sur le travail social juif
}

Historiens et praticiens face aux dossiers personnels

\section{Laure Fourtage}

\section{(2) OpenEdition \\ 1 Journals}

\section{Édition électronique}

URL : http://journals.openedition.org/hommesmigrations/3515

DOI : 10.4000/hommesmigrations.3515

ISSN : 2262-3353

\section{Éditeur}

Musée national de l'histoire de l'immigration

\section{Édition imprimée}

Date de publication : 1 octobre 2015

Pagination : 130-132

ISBN : 978-2-919040-33-9

ISSN : $1142-852 X$

\section{Référence électronique}

Laure Fourtage, «Regards croisés sur le travail social juif », Hommes \& migrations [En ligne], 1312 |

2015, mis en ligne le 31 mai 2016, consulté le 15 septembre 2020. URL : http://

journals.openedition.org/hommesmigrations/3515 


\section{REGARDS CROISÉS SUR LE TRAVAIL SOCIAL JUIF HISTORIENS ET PRATICIENS FACE AUX DOSSIERS PERSONNELS}

Introduction par LAURE FOURTAGE, doctorante en histoire contemporaine, université Paris-I Panthéon-Sorbonne.

$\grave{A}$ travers la présentation de dossiers personnels issus de deux institutions juives d'aide sociale, notre ambition est de montrer l'intérêt, pour les historiens des migrations et du travail social, d'une prise en considération de la documentation produite par les organismes privés de secours. L'historiographie de l'immigration en France s'est en effet essentiellement focalisée sur les politiques publiques et donc vers les sources produites par les autorités administratives concernées. Cette publication s'inscrit dans la continuité de rencontres organisées par Laure Politis, responsable du service Archives, histoire et communication scientifique de la Fondation Casip-Cojasor en 2012-2013. Son originalité réside dans le croisement des regards d'historiens ${ }^{2}$ et de praticiens ${ }^{3}$ sur un même matériau. II s'agit pour chaque vague migratoire d'étudier à deux voix des dossiers d'individus ou de familles pris en charge entre 1945 et 1975 par le Comité juif d'action sociale et de reconstruction (Cojasor) ou le Comité de bienfaisance israélite de Paris (Cbip), devenu en 1963 le Comité d'action sociale israélite de Paris (Casip). Cette période est caractérisée par l'arrivée en France de populations juives d'origines diverses qui font l'objet de l'analyse : les survivants de la Shoah d'Europe centrale et orientale dans l'après-Seconde Guerre mondiale (dossiers de Melle $U$ et de la famille S), les réfugiés d'Égypte arrivés massivement en 1956-1957 (dossiers des familles $K$ et $D$ ) et enfin les juifs de Tunisie venus par vagues successives entre la fin des années 1940 et les années 1970 (dossiers des familles $B$ et $L^{4}$ ).

\section{La France, pays d'accueil ${ }^{5}$ ?}

À eux seuls, les dossiers ne permettent pas de savoir précisément ce qui a poussé ces populations à migrer vers la France, mais l'antisémitisme est souvent l'un des facteurs de leur départ. En effet, la capitulation de l'Allemagne en mai 1945 ne signifie pas pour autant la fin des

\footnotetext{
1. La Fondation CC est née en 2000 de la fusion du Casip (successeur du CBIP créé en 1809) et du Cojasor fondé en 1945. 2. Alexandre De Aranjo, Laura Hobson Faure, Laure Fourtage, Martin Messika, Mônica Raisa-Schpun, Jean-Michel Rallières, Colette Zytnicki. 3. Orly Knafo, Claudine Salamon, Michèle Taieb, Véronique Voisin. 4. Ces dossiers sont conservés à la Fondation CC pour ceux du Cbip et Casip (B : 7758, L:17976) et au Mémorial de la Shoah pour ceux du Cojasor (U : 886, S : 33764, K : 42512, $\mathrm{D}$ : 45053). 5. Ces développements synthétisent les 3 contributions en les mettant en relation avec les résultats d'un entretien réalisé le 6 juillet 2015 avec les assistantes sociales de la fondation sur leurs pratiques actuelles.
} 
persécutions, en particulier en Pologne, Hongrie et Roumanie où des pogroms sont encore perpétrés. En Égypte, l'arrivée au pouvoir de Nasser en 1956 s'accompagne de mesures antisémites (séquestration des biens, arrestations, dénaturalisations). Quant aux pays du Maghreb, le processus de décolonisation engendre parfois des conflits entre les populations juive et musulmane. Des raisons économiques peuvent bien sûr également intervenir, par exemple chez les familles tunisiennes d'artisans et de commerçants qui perdent progressivement leur clientèle, toujours plus nombreuses à migrer. Pour certains migrants, la France représente l'espoir d'une vie plus heureuse. Mais, pour d'autres, elle n'est perçue au départ que comme un pays de transit vers une destination plus lointaine, sans qu'il leur soit toujours possible de poursuivre leur route une fois en France. C'est le cas de Mme D. venue d'Égypte en 1962 avec ses enfants dans l'idée de rejoindre ensuite sa sœur au Canada. Le projet est toutefois compromis en raison des problèmes de santé de la mère et de la déficience mentale de la fille.

La proximité géographique, l'attachement culturel, mais aussi la connaissance du français et la présence de famille ou d'amis sont autant de facteurs qui peuvent influencer le choix entre passage ou installation en France. L'attitude des gouvernements envers les réfugiés, ainsi que le dispositif de secours existant sont aussi des paramètres importants. Disposées après-guerre à n'octroyer que le séjour temporaire en France aux réfugiés juifs d'Europe centrale et orientale, les autorités françaises acceptent en revanche l'installation sur le sol national des populations juives venues de Tunisie et d'Égypte. À chaque époque, un dispositif d'aide massive est mis en place pour répondre aux nombreux besoins des migrants arrivant avec peu ou pas de ressources. Mais, alors que dans l'immédiat après-guerre les institutions juives de secours en supportent presque tout le poids financier, elles sont progressivement inclues dans des programmes d'assistance associant les pouvoirs publics et des organismes privés non juifs comme le Service social d'aide aux émigrants.

\section{Du secours à l'accompagnement social}

Entre 1945 et 1975, l'approche des travailleurs sociaux à l'égard de leurs usagers a beaucoup évolué : d'une action ne visant qu'à donner des réponses matérielles immédiates à une prise en charge plus globale. La professionnalisation des assistantes sociales passe notamment par l'apprentissage des méthodes américaines du casework, introduites dès la fin des années 1940 dans les institutions juives grâce au Joint. Cette aide individualisée repose sur la prise en compte des facteurs psychologiques, sociaux et environnementaux des dysfonctionnements. Quel que soit le cas toutefois, la raison d'être du suivi repose uniquement sur l'autonomie financière des usagers. Lorsque l'assistante sociale estime que les conditions sont réunies pour que la famille soit autosuffisante, elle met donc fin à la relation. Aujourd'hui, l'approche est inversée. La fondation ne propose plus "un secours", terme considéré comme trop associé à l'idée d'une distribution systématique d'aide financière, mais "un accompagnement social", expression signalant la centralité d'un suivi sans limite de durée. Les aides monétaires trouvent leur place à côté d'un large éventail d'autres aides : éducative, administrative, familiale, etc. Une exception toutefois : l'action menée à l'égard des réfugiés par une "mission" du service social, héritière du Service spécial des immigrants (SSI) créé au sein du Cojasor en 1946. Comme le faisait le SSI, cette "mission" octroie une aide financière régulière aux populations juives étrangères qui, souvent arrivées clandestinement, n'ont pas droit aux aides publiques. Cette prise en charge, coûteuse pour la fondation, ne concerne toutefois qu'un effectif réduit. En 2014, seules 141 familles étaient concernées, soit 2,3\% du total. À titre de 


\section{REPÉRAGE}

comparaison, parmi l'ensemble des individus suivis en 1948, 52,7 \% étaient des immigrés ${ }^{6}$. De plus, elle ne dure en moyenne que trois ans, le temps que la famille soit juridiquement fixée sur son sort. Le dossier est alors transféré au service social général si la famille désire le maintien du suivi.

\section{Entre neutralité et empathie}

Dans tous les dossiers étudiés ici, les notes des assistantes sociales présentent des jugements de valeur sur le physique et le moral des usagers. Il est toutefois impossible de mesurer l'impact réel de ces jugements sur l'octroi du secours. On trouve par exemple "type

Dans tous les dossiers étudiés ici, les notes des assistantes sociales présentent

des jugements de valeur sur le physique et le moral des usagers. Il est toutefois

impossible de mesurer

l'impact réel de ces jugements sur l'octroi du secours. sérieux qui nous fait bonne impression" en parlant de M. K. La relation avec l'assistante paraît ici plutôt sereine, voire très proche dans le cas de Mme D. - trop proche, disent aujourd'hui les travailleurs sociaux de la fondation. En effet, si les assistantes sociales se doivent d'être à l'écoute, Claudine Salamon souligne la nécessité de conserver "une distance suffisante". Orly Knafo parle alors d'une "bienveillance professionnelle". À cela s'ajoute la loi de réforme de l'action sociale de 2002 qui, en donnant l'accès des usagers à leur dossier, impose aux assistantes sociales davantage de retenue dans leurs écrits. Michèle Taieb explique que l'apparence physique, le comportement en entretien ou le cadre de vie continuent d'être pris en considération si un de ces éléments est perçu comme "très caractéristique", mais cela n'apparaît plus dans les dossiers.

\section{Une course à l'intégration}

Dans les cas étudiés, l'assistance est non seulement envisagée comme provisoire par les institutions mais elle se veut la plus courte possible. Excepté le dossier de la famille L. resté ouvert plus de dix-sept ans (avec un suivi discontinu), la durée moyenne de prise en charge est de deux ans et demi. Pour certains cas, la clôture du dossier se fait naturellement : suite au départ au Canada des enfants de Mme $D$. après son décès, suite au remariage de $M m e$ B., ou encore après l'obtention d'un prêt au logement censé résoudre les dernières difficultés matérielles de la famille K. Pour d'autres comme E. U., l'arrêt du suivi se fait brutalement. Quant à la famille L., les troubles mentaux de la mère et leurs effets sur la situation sociale de cette famille ne sont pris en compte que lorsque celle-ci est déjà complètement marginalisée. Jusque-là, l'assistante profère des menaces, celle du placement de l'enfant et celle, présente dans d'autres dossiers et mise à exécution ici, de l'arrêt du secours financier. Pour Véronique Voisin, "c'était le meilleur moyen pour créer une rupture, une fin de non-recevoir, de précipiter les choses et de mettre fin à cet accompagnement". Aujourd'hui, la meilleure approche envers les personnes sans domicile fixe pose toujours question mais l'attitude des travailleurs sociaux a changé : conscients que ce contact avec la "communauté" représente parfois le dernier lien avec la société, ils tentent de le maintenir. Pour 4 dossiers, on sait que les personnes ont finalement quitté la France quelques années après la fin du suivi. La raison est inconnue mais soulignons toutefois que la ré-émigration est souvent envisagée par les usagers comme une solution à leurs difficultés d'intégration en France. 